\title{
Assessment of humoral immune response of a Cytomegalovirus DNA- vaccine candidate in BALB/c mice
}

\author{
Vahabpour $\mathbf{R}^{1}$, Aghasadeghi MR ${ }^{1}$, Goudarzifar $\mathbf{F}^{2}$, Keyvani $\mathbf{H}^{3}$, Ataei- Pirkooh $\mathbf{A}^{3}$, Monavari $\mathbf{S H}^{3 *}, \mathbf{E s l a m i}^{4,5}$ \\ ${ }^{1}$ Department of Hepatitis and AIDS, Pasteur Institute of Iran, Tehran, Iran \\ ${ }^{2}$ Department of Virology, Iran University of Medical Sciences, International Campus, Tehran, Iran \\ ${ }^{3}$ Department of Virology, Iran University of Medical Sciences, International Campus, Tehran, Iran. \\ ${ }^{4}$ Department of Genetics, Tehran Medical Sciences Branch, Islamic Azad University, Tehran, Iran. \\ ${ }^{5}$ Applied Biotechnology Research Center, Tehran Medical Sciences Branch, Islamic Azad University, Tehran, Iran.
}

\begin{abstract}
Introduction: Glycoprotein $\mathrm{B}(\mathrm{gB})$ is the major antigen for induction of humoral responses against human cytomegalovirus (HCMV) making it an attractive candidate for immune prophylaxis. In the present study, the humoral immune response of BALB/c mice to a truncated HCMV gB protein fused with GFP was evaluated. Methods: The truncated $\mathrm{gB}$ coding sequence was synthesized and cloned in pEGFPN1 eukaryotic expression vector and expressed in HEK 293T cell line. After optimization, expression of the recombinant truncated HCMV gB was verified using HRPconjugated polyclonal antibody specific for HCMV gB.The level of humoral immune responses was assessed in mice using DNA/DNA, peptide/peptide, and DNA/ peptide (prime-boost) immunization strategies. Results: Cloning of the truncated $\mathrm{gB}$ coding sequence in the pEGFPN1 was verified by restriction enzyme analysis and sequencing. After optimizing the transfection procedure the number of the GFP positive cells reached $32 \%$. Western blot analysis confirmed the in vitro expression of the truncated HCMV $\mathrm{gB}$ protein with an apparent molecular weight of approximately $70 \mathrm{kDa}$. In vivo prime-boost immunization using HCMV gB DNA/peptide regimen showed significantly higher humoral immune responses compared to the control groups. Conclusion: This study demonstrated that the pEGFPN1 eukaryotic expression vector could be used to optimize and evaluate the expression of this truncated protein.The results also showed that the DNA/peptide vaccination could induce a significant antibody response in animal model.
\end{abstract}

KEYWORDS: human cytomegalovirus, Glycoprotein B, DNA-based vaccine.

\section{INTRODUCTION}

Human cytomegalovirus (HCMV) is a universally distributed virus belonging to beta- subfamily of the enveloped, doublestranded DNA herpes viruses causing asymptomatic latent lifelong infections in $60-90 \%$ of world population $[1,2]$. Although infections with $\mathrm{HMCV}$ in immunocompetent hosts are relatively benign, but have severe consequences in immunocompromized individuals resulting in high mortality and morbidity [1,2]. Recipient of solid organ transplants and AIDS patients do not control the infection well with virus infecting various cells types resulting in life-threatening tissue invasive disease and blindness [3]. Furthermore, in utero

\footnotetext{
*Corresponding Author: Seyed Hamidreza Monavari, Ph.D; Department of Virology, Iran University of Medical Sciences, International Campus, Tehran, Iran.

Email: hrmonavari@yahoo.com

Tel/Fax: (+98) 2188602205
}

infection of the immunologically immature fetus through primary infection or reactivation of a latent virus is an important cause of congenital defects such as loss of vision or hearing, mental retardation and cerebral palsy [4]. Although maternal seroprevalence, which has been estimated to range from $84-100 \%$ in developing countries, has been related to the incidence of fetus infection, but the burden of the disease in developing countries is unknown due to the lack of data on sequelae $[5,6]$. In addition, an association between subclinical infection with HCMV and immune senescence, atherosclerosis, and various forms of cancer such as medulloblastoma, malignant glioblastoma, EBV-negative Hodgkin's lymphoma, prostatic carcinoma, colon and breast cancer has been proposed $[1,7]$. However, despite the urgent need for prophylactic and therapeutic vaccines for HCMV, no candidate vaccines are under consideration for licensure [8]. Research in this area has focused on either attenuation of the live virus or the use of 
individual immune-dominant antigens as purified proteins, DNA or vectored vaccines [8].

CMV is a large virus with a $230 \mathrm{~kb}$ double-stranded DNA genome coding for approximately 200 proteins of which UL55 (also known as glycoprotein B) has been recognized as a key immunogen eliciting strong immune responses in humans and a target for vaccine development $[8,9]$. Antibody production is considered a major defense against viruses and HCMV antiglycoprotein B (anti gB) is detected in all infected individuals. $\mathrm{gB}$ is a highly conserved, 906 amino-acid long protein consisting of an ectodomain, a hydrophobic region, a transmembrane and a cytoplasmic domain which is required for viral entry into all cell types including placental precursor cells and cell to cell spread of the virus [8-10]. Several antibodybinding sites have been identified on $\mathrm{gB}$ of which two domains containing three antigenic sites are capable of eliciting neutralizing antibodies during natural infection [9-12]. The gB region also contains several non-linear or assembled epitopes [9-12] and of the 5 antigenic domains identified on this protein, 4 have mapped to the first $693 \mathrm{aa}$ of the ectodomain, identifying the extracellular portion of HCMV $\mathrm{gB}$ as the immunodominant target for elicitation of neutralizing antibodies following natural infection [9-12]. In the present study the coding sequence for this fragment was synthesized and cloned as a DNA-based vaccine expressing truncated $\mathrm{HCMV} \mathrm{gB}$ and its antigenicity was assessed in BALB/c mice.

\section{MATERIALS and METHODS}

\section{Construction of recombinant plasmid and peptide synthesis}

The coding sequence for the ectodomain of HCMV glycoprotein B (accession number EF531305) containing sites for restriction enzymes Hind III and Sac I was chemically synthesized (Biomatik, Canada) and a cellular protease site (GCA GCA TAC), was added to the 3 'end of the sequence for proteolytic digestion of the recombinant protein after expression in mammalian cells. pUC57containing truncated sequence coding for amino acids 1-693was double digested and the eluted fragment was subcloned in a similarly double digested pEGFPN1 plasmid downstream of CMV promoter and immediately upstream of EGFP start codon. The construct was validated by restriction enzyme analysis and was commercially sequenced (SeqLab, Germany). Plasmid EGFPN1 containing fused $\triangle \mathrm{gB}:$ :egfp gene was purified using Endo-free plasmid Midi kit (Qiagen, Germany). Peptide fragment "DSYHFSSAKMTATFLSKKQEVN" corresponding to aa 361-383 covering a part of the discontinuous epitope of antigenic domain II was also chemically synthesized (Biomatik,
Canada) for evaluation of the immune response of the vaccinated mice and for immunization.

\section{Expression of recombinant of $\triangle \mathrm{gB}$ :EGF protein in HEK 293T cells}

Human embryonic kidney HEK 293T cell line (Pasteur Institute of Iran) was used for expression of the truncated HCMV gB fused to EGFP by transfection of the cell line with the purified recombinant pEGFPN1 plasmid containing the fused gene using Polyfect reagent (Qiagen, USA) according to the manufacturer's instructions. Cells were maintained in DMEM (Life Technology, USA), supplemented with $10 \%$ fetal bovine serum (Life Technology, USA), glutamine $(2 \mathrm{mM})$, penicillin $\mathrm{G}$ $(100 \mathrm{U} / \mathrm{ml})$, streptomycin $(100 \mathrm{mg} / \mathrm{ml})$ in $5 \% \mathrm{CO} 2$ and $37^{\circ} \mathrm{C}$.

\section{Quantification of protein expression by flowcytometry}

In order to determine the transfection efficiency and expression level of the fused gene flow cytometry was used taking advantage of GFP marker that had been fused to the C-terminal of truncated HCMV gB protein. Briefly, $48 \mathrm{~h}$ post-transfection HEK 293T cells were harvested after treatment with trypsin/EDTA and resuspended in $1 \mathrm{ml}$ of phosphate buffer saline (PBS pH 7.5) and directly applied to Partec PAS flow cytometer (Partec, Germany) using a bivariate scatter plot of fluorescence versus forward scatter, by gate setting with untransfected cells. Approximately 20,000 events from the transfected cell population per sample were analyzed.

Analysis of protein expression by Western blot

Transfected HEK 293T cells were pelleted after trypsinization and washed three times with PBS ( $\mathrm{pH} 7.5)$. Cell pellet was lysed in $200 \mu \mathrm{l}$ of extraction buffer containing $50 \mathrm{mM}$ Tris- $\mathrm{HCl}$ (pH 7.5), $50 \mathrm{mM} \mathrm{NaCl}, 1 \mathrm{mM}$ EDTA, $0.5 \% \mathrm{NP}-40$ and $0.5 \%$ SDS and used for SDS-PAGE analysis. Electrophoreticallyseparated protein bands were transferred to nitrocellulose membrane (Bio-Rad, USA) and skim milk blocked membranes (5\% milk in PBS-Tween 20) were incubated with 1:5000 dilution of goat polyclonal anti gb-HRP (Abcam, USA) for 60 min at room temperature. Antigen-antibody reaction was visualized using DAB (3, 3'-diaminobenzidine tetrahydrochloride (BioRad, USA).

\section{Animals and immunization schedule}

Inbred BALB/c female mice, age-averaged and $20 \mathrm{~g}$ weight each were obtained from Pasteur Institute of Iran and handled according to the animal care ethics (Declaration of Helsinki, Fifth revision 2000). Mice were divided into five groups of 6 and were immunized subcutaneously on days 0,14 and 21 receiving DNA/DNA (G1), DNA/peptide (G2), and peptide/peptide (G3) injections as listed in Table 1. Synthetic peptide was used at a concentration of $20 \mu \mathrm{g}$ emulsified with Montanide ISA720 (M720) at a ratio of 8:2. The control groups were injected with pEGFPN1 plasmid without the $\mathrm{gB}$ insert (G4) and PBS (G5).

Table 1. Immunization schedule of BALB/c mice with truncated $\mathrm{gB}$ and a synthetic $\mathrm{gB}$ epitope

\begin{tabular}{|c|c|c|c|c|}
\hline Groups & Regimen & Day 0 (Prime) & Day 14(booster) & Day 21(booster) \\
\hline G1 & DNA/DNA & pgB-EGFP $(50 \mu \mathrm{g})$ & - & pgB-EGFP $(50 \mu \mathrm{g})$ \\
\hline G2 & DNA/Peptide/Peptide & pgB-EGFP $(50 \mu \mathrm{g})$ & gB peptide $(10 \mu \mathrm{g})$ & gB peptide $(10 \mu \mathrm{g})$ \\
\hline G3 & Peptide/Peptide/Peptide & gB peptide $(10 \mu \mathrm{g})$ & gB peptide $(10 \mu \mathrm{g})$ & gB peptide $(10 \mu \mathrm{g})$ \\
\hline G4 & DNA & pEGFPN1 $(50 \mu \mathrm{g})$ & - & pEGFPN1 $(50 \mu \mathrm{g})$ \\
\hline G5 & PBS & PBS & - & PBS \\
\hline
\end{tabular}

\section{Enzyme-Linked Immunosorbent Assay (ELISA)}

Humoral immune response of immunized mice was quantified by ELISA assay. Briefly, synthetic HCMV gB peptide $(10 \mu \mathrm{g} /$ $\mathrm{ml})$ was used as the capture molecule to coat 96-well micro plates (Nunc, Denmark). After washing and blocking steps, the sera were diluted 1:50 in the blocking solution, applied in duplicate, and incubated at $37{ }^{\circ} \mathrm{C}$ for $1 \mathrm{hr}$. The plates were then washed three times with PBS-Tween solution and further 
incubated with 1:10000 dilution of HRP-labeled goat antimouse IgG (Abcam, USA). Color was developed by adding 100 $\mu \mathrm{l}$ of tetramethylbenzidine (TMB) substrate (Abcam, USA) to each well and absorbance was measured at $450 \mathrm{~nm}$.

\section{Statistical Analyses}

All experiments were performed in triplicate or repeated three times. The differences between experimental groups were statistically analyzed by Mann-Whitney non-parametric test and one-way ANOVA. P values less than $0.05(p<0.05)$ were considered as significant.

\section{RESULTS}

\section{Construction of recombinant pN1gB::EGFP}
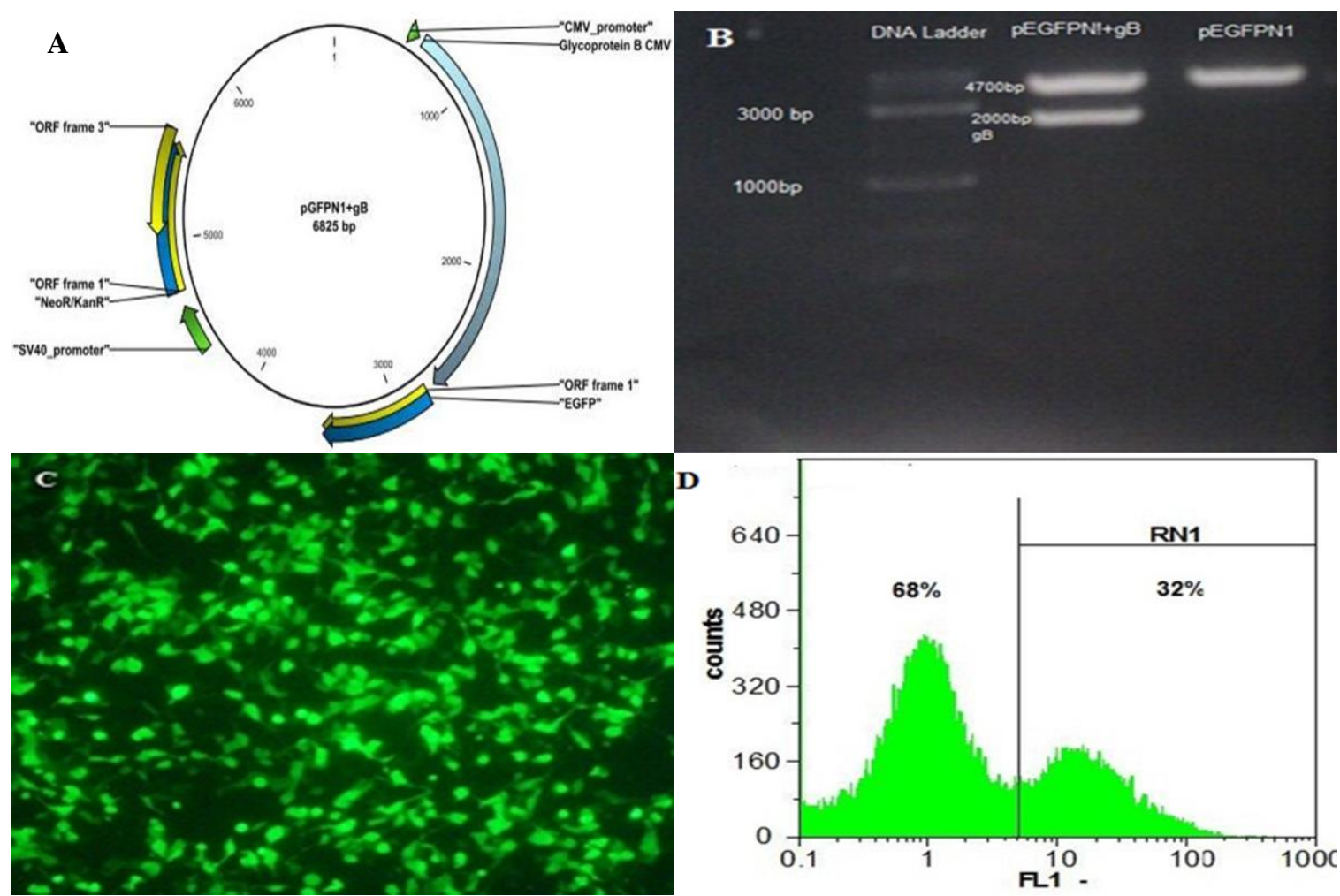

Fig.1. (A) Schematic representation of the $\mathrm{p} \Delta \mathrm{gB}$-EGFP construct (B) Digestion pattern of recombinant $\mathrm{p} \Delta \mathrm{gB}$-EGFP (C) Immunofluorescennt microscopy image of the cells transfected with $\mathrm{p} \Delta \mathrm{gB}$-EGFP construct expressing GFP $(\times 40)$; (D) Flow-cytometry plot showing the high fluorescent intensity of transfected cells (RN1) compared to un-transfected cells. Transfection rate was calculated as the percentage of the GFP positive cells (32\%) to the total number of cells in the gated region.

\section{Analysis of recombinant protein expression by Western blot}

Immunoblot analysis of the lysates of pN1gB-EGFP transfected cells showed the in vitro expression of the HCMV gB fusion

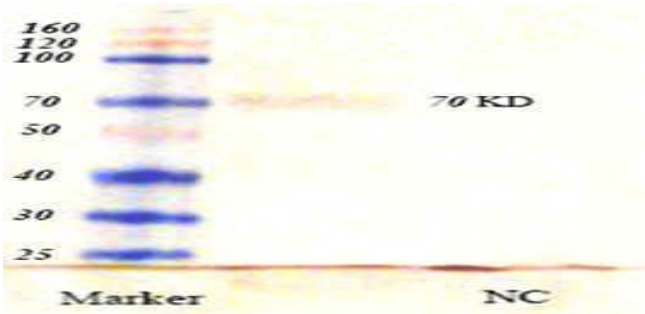

Fig.2. Western blot result of the truncated gB expressed in HEK 293T cell line using 1:5000 dilution of goat polyclonal anti gb-HRP.
Subcloning of the truncated $\mathrm{gB}$ in pEGFPN1 plasmid downstream of CMV promoter was confirmed by restriction enzyme digestion (Fig.1A and 1B) and fusion of $\Delta g B$ with $e g f p$ gene was verified by sequencing. The $\mathrm{N}$-terminal signal sequence of $\mathrm{gB}$ was retained to facilitate secretion of the expressed protein.

\section{Evaluation of transfection efficiency and expression}

Quantification of the GFP positive cells was performed using Partec PAS flow cytometer. Untransfected cells were used as control to set the gates and the gated region was analyzed for GFP positive cells at $488-508 \mathrm{~nm}$. The percentage of the cells expressing GFP in the gated region was $32 \%$ after transfection optimization (Fig.1C \&1D). 


\section{Mice humoral Response}

The HCMVgB-specific humoral responses of mice were analyzed by ELISA 3 weeks after the last immunization. Total IgG antibody response to HCMV gB epitope is shown in Figure 3 .

The anti-HCMVgB IgG levels in G1-G3 test groups were significantly higher than the control groups $(p<0.05)$. The antiHCMVgB antibody in DNA/Peptide/Peptide group was however, significantly higher than anti-gB antibodies generated by other test groups.

\section{DISCUSSION}

The present study provides the preclinical evaluation of hummoral immune responses to a candidate HCMV DNAbased vaccine using DNA/DNA, peptide/peptide and DNA/peptide immunization strategies in a mouse model. In order to create the DNA-based candidate vaccine the truncated sequence for the ectodomain of HCMV glycoprotein B coding for amino acids 1-693, was cloned in pEGFPN1 plasmid downstream of CMV promoter and immediately upstream of EGFP start codon. HCMV glycoprotein B is the dominant antigen for the induction of humoral immunity and the extracellular part of the protein contains the majority of the antigenic structures for the humoral immune response against CMV [13]. Therefore, search for the active and passive immuno-prophylactic candidates against the virus has been focused mainly on the use of this antigen [14]. Previous studies have characterized extracellular portion of HCMV $\mathrm{gB}$ as a complex antibody-binding domain consisting of several epitopes and two antibody-binding sites located between aa 5078 and 552-635 have been identified as the target of neutralizing antibodies in the sera from persons naturally infected with hCMV $[15,16]$.

One of the vaccine candidate that is currently in clinical trial is a DNA vaccine consisting of two plasmids expressing pp65 and glycoprotein B in apoloxamer-based formulation. In the this study $\mathrm{gB}$ antibody titer was detected in $17-25 \%$ of seronegative recipients but, the vaccine did not boost gB-specific antibody titers in seropositive vaccinees more than four-fold [17]. Another DNA vaccine candidate that was formulated with PBS instead of poloxamer and with an additional plasmid encoding IE1 in addition to the $\mathrm{gB}$ and pp65-encoding plasmids, was evaluated with the live Towne vaccine in a prime-boost regimen [18]. This vaccine was poorly immunogenic and regardless of route of administration, no more than $15 \%$ of the vaccines in any of the groups responded to the vaccine. In the current report, we applied a novel prime-boost approach in which the priming injections consisted of HCMV gB-GFP fusion DNA alone $(50 \mu \mathrm{g})$, followed by HCMV gB synthetic peptide emulsified with Montanide. Analysis of the immune responses indicated that the prime-boost immunization induces significant humoral responses 3 weeks after the third injection. However, all three strategies could generate IgG against HCMV gB coated antigens compared to the control groups. Our data showed that priming with DNA (pgB-EGFP) followed by gB peptide as a booster effectively stimulate hummoral immunity in mice. Our results promisingly demonstrated that this DNA/peptide vaccination regimen in contrast with previous bivalent HCMV DNA vaccine could induce a stable antibody response [18]. Our results further confirmed that for DNA vaccination modality prime/boost regimen is among the most effective approaches for eliciting protective antibodies [3].

Results from the current study indicate that a DNA vaccine with the component expressing the extracellular domain of HCMV glycoprotein B might be a suitable candidate for further development and clinical evaluation. However, the cellular immune responses to the proposed antigen remains to be evaluated and further studies are required to improve the stability and durability of the immune responses of this candidate vaccine.

\section{ACKNOWLEDGEMENT}

This work was supported by a grant from the Ministry of Health and Medical Education.

\section{CONFLICT OF INTEREST}

The authors declare that they have no conflict of interest.

\section{REFERENCES}

1. Wu SJ, Villarreal DO, Shedlock DJ, Weiner DB. Synthetic DNA approach to cytomegalovirus vaccine/immune therapy. Advances in experimental medicine and biology. 2015;848:131-48. doi:10.1007/978-14939-2432-5 7.

2. Vanarsdall AL, Johnson DC. Human cytomegalovirus entry into cells. $\begin{array}{lll}\text { Current opinion in virology. 2012;2(1):37-42. } & \text {. }\end{array}$ doi:10.1016/j.coviro.2012.01.001.

3. Kirchmeier M, Fluckiger AC, Soare C, Bozic J, Ontsouka B, Ahmed T et al. Enveloped virus-like particle expression of human cytomegalovirus glycoprotein B antigen induces antibodies with potent and broad neutralizing activity. Clinical and vaccine immunology : CVI 2014;21(2):174-80. doi:10.1128/CVI.00662-13.

4. Hartikka J, Bozoukova V, Morrow J, Rusalov D, Shlapobersky M, Wei Q et al. Preclinical evaluation of the immunogenicity and safety of plasmid DNA-based prophylactic vaccines for human cytomegalovirus. Human vaccines \& immunotherapeutics. 2012;8(11):1595-606. doi: $10.4161 /$ hv. 21225 .

5. Lanzieri TM, Dollard SC, Bialek SR, Grosse SD. Systematic review of the birth prevalence of congenital cytomegalovirus infection in developing countries. International journal of infectious diseases : IJID : official publication of the International Society for Infectious Diseases. 2014;22:44 8. doi:10.1016/j.ijid.2013.12.010

6. Manicklal S, Emery VC, Lazzarotto T, Boppana SB, Gupta RK. The "silent" global burden of congenital cytomegalovirus. Clinical microbiology reviews. 2013;26(1):86-102. doi:10.1128/CMR.00062-12.

7. Kabanova A, Perez L, Lilleri D, Marcandalli J, Agatic G, Becattini S et al. Antibody-driven design of a human cytomegalovirus gHgLpUL128L subunit vaccine that selectively elicits potent neutralizing antibodies. Proceedings of the National Academy of Sciences of the United States of America. 2014;111(50):17965-70. doi:10.1073/pnas.1415310111.

8. Schleiss MR. Developing a Vaccine against Congenital Cytomegalovirus (CMV) Infection: What Have We Learned from Animal Models? Where Should We Go Next? Future virology. 2013;8(12):1161-82. doi:10.2217/fvl.13.106.

9. Wang H, Huang C, Dong J, Yao Y, Xie Z, Liu X et al. Complete protection of mice against lethal murine cytomegalovirus challenge by immunization with DNA vaccines encoding envelope glycoprotein complex III antigens $\mathrm{gH}, \mathrm{gL}$ and gO. PloS one. 2015;10(3):e0119964. doi:10.1371/journal.pone.0119964.

10. Burke HG, Heldwein EE. Crystal Structure of the Human Cytomegalovirus Glycoprotein B. PLoS pathogens. 2015;11(10):e1005227. doi:10.1371/journal.ppat.1005227.

11. Kniess N, Mach M, Fay J, Britt WJ. Distribution of linear antigenic sites on glycoprotein gp55 of human cytomegalovirus. Journal of virology. 1991;65(1):138-46

12. Potzsch S, Spindler N, Wiegers AK, Fisch T, Rucker P, Sticht H et al. B Cell Repertoire Analysis Identifies New Antigenic Domains on Glycoprotein B of Human Cytomegalovirus which Are Target of 
Neutralizing Antibodies. PLoS pathogens. 2011;7(8). doi:ARTN e1002172 10.1371/journal.ppat.1002172.

13. Werner BG, Snydman DR, Freeman R, Rohrer R, Tilney NL, Kirkman RL. Cytomegalovirus immune globulin for the prevention of primary CMV disease in renal transplant patients: analysis of usage under treatment IND status. Transplant proc 1993; 25:1441-3.

14. Schoppel K, Hassfurther E, Britt W, Ohlin M, Borrebaeck CA, Mach M. Antibodies specific for the antigenic domain 1 of glycoprotein B (gpUL55) of human cytomegalovirus bind to different substructures. Virology. 1996; 216:133-45. doi:10.1006/viro.1996.0040.

15. Meyer H, Sundqvist V.A, Pereira L and Mach M. Glycoprotein gp116 of human cytomegalovirus contains epitopes for strain-common an strain- specific antibodies. J. Gen. Virol. 1992; 73:2375-83.

16. Wagner B, Kropff B, Kalbacher H, Britt W, Sundqvist VA, Ostberg L et al. A continuous sequence of more than 70 amino acids is essential for antibody binding to the dominant antigenic site of glycoprotein gp58 of human cytomegalovirus. J. Virol. 1992; 66:5290-7.

17. Selinsky C, Luke C, Wloch M, Geall A, Hermanson G, Kaslow D et al. A DNA-based vaccine for the prevention of human cytomegalovirusassociated diseases. Human vaccines. 2005;1(1):16-23.

18. Jacobson MA, Adler SP, Sinclair E, Black D, Smith A, Chu A et al. A CMV DNA vaccine primes for memory immune responses to liveattenuated CMV (Towne strain). Vaccine. 2009;27(10):1540-8. doi:10.1016/j.vaccine.2009.01.006. 